\title{
Adversarial Network Compression
}

\author{
Vasileios Belagiannis $^{\star 1}$, Azade Farshad ${ }^{\star 1,2}$, Fabio Galasso ${ }^{1}$ \\ 1 Innovation OSRAM GmbH, Garching b. München, Germany \\ 2 Technische Universität München, Garching b. München, Germany
}

\begin{abstract}
Neural network compression has recently received much attention due to the computational requirements of modern deep models. In this work, our objective is to transfer knowledge from a deep and accurate model to a smaller one. Our contributions are threefold: (i) we propose an adversarial network compression approach to train the small student network to mimic the large teacher, without the need for labels during training; (ii) we introduce a regularization scheme to prevent a trivially-strong discriminator without reducing the network capacity and (iii) our approach generalizes on different teacher-student models.

In an extensive evaluation on five standard datasets, we show that our student has small accuracy drop, achieves better performance than other knowledge transfer approaches and it surpasses the performance of the same network trained with labels. In addition, we demonstrate state-ofthe-art results compared to other compression strategies.
\end{abstract}

\section{Introduction}

Deep learning approaches dominate on most recognition tasks nowadays. Convolutional Neural Networks (ConvNets) rank highest on classification [56], object detection [35, image segmentation [6] and pose estimation [38, just to name a few examples. However, the superior performance comes at the cost of model complexity and large hardware requirements. Consequently, deep models often struggle to achieve real-time inference and cannot generally be deployed on resource-constrained devices, such as mobile phones.

In this work, our objective is to compress a large and complex deep network to smaller one. Network compression is a solution that only recently attracted more attention because of the deep neural networks. One can train a deep model with quantized or binarized parameters [484154, factorize it, prune network connections [16]26 or transfer knowledge to a small network 2|420. In the latter case, the student network is trained with the aid of the teacher.

We present a network compression algorithm whereby we complement the knowledge transfer, in the teacher-student paradigm, with adversarial training. In our method, a large and accurate teacher ConvNet is trained in advance. Then, a small student ConvNet is trained to mimic the teacher, i.e. to obtain the same output. Our novelty lies in drawing inspiration from Generative Adversarial Networks (GANs) [14 to align the teacher-student distributions. We propose a

\footnotetext{
* Equal contribution.
} 
two-player game, where the discriminator distinguishes whether the input comes from the teacher or student, thus effectively pushing the two distributions close to each other. In addition, we come up with a regularization scheme to help the student in competing with the discriminator. Our method does not require labels, only the discriminator's objectives and an L2 loss between the teacher and student output. We name our new algorithm adversarial network compression.

An extensive evaluation on CIFAR-10 [27], CIFAR-100 [27], SVHN [37, Fashion-MNIST [55] and ImageNet [10] shows that our student network has small accuracy drop and achieves better performance than the related approaches on knowledge transfer. In addition, we constantly observe that our student achieves better accuracy than the same network trained with supervision (i.e. labels). In our comparisons, we demonstrate superior performance next to other compression approaches. Finally, we employ three teacher and three student architectures to support our claim for generalization.

We make the following contributions: (i) a knowledge transfer method based on adversarial learning to bridge the performance of a large model with a smaller one with limited capacity, without requiring labels during learning; (ii) a regularization scheme to prevent a trivially-strong discriminator and (iii) generalization on different teacher-student architectures.

\section{Related Work}

Neural network compression has been known since the early work of [17/49, but recently received much attention due to the combined growth of performance and computational requirements in modern deep models. Our work mostly relates to model compression [2/4 and network distillation [20. We review the related approaches on neural network compression by defining five main categories and then discuss adversarial training.

I. Quantization $\&$ Binarization The standard way to reduce the size and accelerate the inference is to use weights with discrete values [48,54. The Trained Ternary Quantization [65] reduces the precision of the network weights to ternary values. In incremental network quantization [64, the goal is to convert progressively a pre-trained full-precision ConvNet to a low-precision. Based on the same idea, Gong et al. [13] have clustered the weights using k-means and then performed quantization. The quantization can be efficiently reduced up to binary level as in XNOR-Net [41, where the weight values are -1 and 1, and in BinaryConnect [9], which binarizes the weights during the forward and backward passes but not during the parameters' update. Similar to binary approaches, ternary weights $(-1,0,1)$ have been employed as well [31.

II. Pruning Reducing the model size (memory and storage) is also the goal of pruning by removing network connections [515160]. At the same time, it prevents over-fitting. In [16], the unimportant connections of the network are pruned and the remaining network is fine-tuned. Han et al. [15] have combined 
the idea of quantization with pruning to further reduce the storage requirements and network computations. In HashedNets [7, the network connections have been randomly grouped into hash buckets where all connections of the same bucket share the weight. However, the sparse connections do not necessarily accelerate the inference when employing ConvNets. For this reason, Li et al. 32] have pruned complete filters instead of individual connections. Consequently, the pruned network still operates with dense matrix multiplications and it does not require sparse convolution libraries. Parameter sharing has also contributed to reduce the network parameters in neural networks with repetitive patterns [3 46].

III. Decomposition / Factorization In this case, the main idea is to construct low rank basis of filters. For instance, Jaderberg et al. [26] have proposed an agnostic approach to have rank-1 filters in the spatial domain. Related approaches have also explored the same principle of finding a low-rank approximation for the convolutional layers [3/1129/58. More recently, it has been proposed to use depthwise separable convolutions, as well as, pointwise convolutions to reduce the parameters of the network. For example, MobileNets are based on depthwise separable convolutions, followed by pointwise convolutions [21. In a similar way, ShuffleNet is based on depthwise convolutions and pointwise group convolutions, but it shuffles feature channels for increased robustness [63.

IV. Efficient Network Design The most widely employed deep models, AlexNet [28] and VGG16 [47, demand large computational resources. This has motivated more efficient architectures such as the Residual Networks (ResNets) [18] and their variants 2361, which reduced the parameters, but maintained (or improved) the performance. SqueezeNet 24 trims the parameters further by replacing $3 \times 3$ filters with $1 \times 1$ filters and decreasing the number of channels for $3 \times 3$ filters. Other recent architectures such as Inception [50, Xception [8], CondenseNet [22] and ResNeXt [56] have also been efficiently designed to allow deeper and wider networks without introducing more parameters than AlexNet and VGG16. Among the recent architectures, we pick ResNet as the standard model to build the teacher and student. The reason is the model simplicity where our approach applies to ResNet variations as well as other architectures.

Network compression categories I-IV address the problem by reducing the network parameters, changing the network structure or designing computationally efficient components. By contrast, we focus on knowledge transfer from a complex to a simpler network without interventions on the architecture. Our knowledge transfer approach is closer to network distillation, but it has important differences that we discuss bellow.

$V$. Distillation Knowledge transfer has been successfully accomplished in the past 2 233, but it has been popularized by Hinton et al. 20]. The goal is to transfer knowledge from the teacher to the student by using the output before the softmax function (logits) or after it (soft targets). This task is known as network compression [2]4 and distillation [2040]. In our work, we explore the problem for recent ConvNet architectures for the teacher and student roles. We 
demonstrate that network compression performs well with deep models, similar to the findings of Urban et al. [53. Differently from the earlier works, we introduce adversarial learning into compression for the first time, as a tool for transferring knowledge from the teacher to the student by their cooperative exploration. Also differently from the original idea of Hinton et al. [20] and the recent one from $\mathrm{Xu}$ et al. [57, we do not require labels for the student training during compression. In the experiments, our results are constantly better than network distillation.

Adversarial Learning Our work is related to the Generative Adversarial Networks (GAN) [14 where a network learns to generate images with adversarial learning, i.e. learning to generate images which cannot be distinguished by a discriminator network. We take inspiration from GANs and introduce adversarial learning in model compression by challenging the student's output to become identical to the teacher. Closer to our objective is the work from Isola et al. 25] to map an image to another modality with a conditional Generative Adversarial Network (cGAN) 36. Although, we do not have a generator in our model, we aim to map the student to teacher output given the same input image. However, compared to the teacher, the student is a model with limited capacity. This motivates a number of novel contributions, needed for the successful adversarial training.

\section{Method}

We propose the adversarial network compression, a new approach to transfer knowledge between two networks. In this section, we define the problem and discuss our approach.

\subsection{Knowledge Transfer}

We define a deep and accurate network as teacher $f_{t}\left(x ; w_{t}\right)$ and a small network as student $f_{s}\left(x ; w_{s}\right)$. The teacher has very large capacity and is trained on labeled data. The student is a shallower network with significant less parameters. Both networks perform the same task, given an input image $x$. Our objective is to train the student to mimic the teacher by predicting the same output. To achieve it, we introduce the discriminator $D$, another network that learns to detect the teacher / student output based on adversarial training. We train the student together with $D$ by using the knowledge of the teacher for supervision.

In this work, we address the problem of classification. For transferring knowledge, we consider the unscaled log-probability values (i.e. logits) before the softmax activation function, as well as, features from earlier layers. Bellow, we simplify the notation to $f_{t}(x)$ for the teacher and $f_{s}(x)$ for the student network output (logits). In addition, the feature representation of the teacher at $k-t h$ layer is defined as $f_{t}^{k}(x)$ and for the student at $l$ - th layer is denoted as $f_{s}^{l}(x)$. In practice, $f_{t}^{k}(x)$ and $f_{s}^{l}(x)$ are the last layers before the logits. An overview of our method is illustrated in Fig. 1 . 




Fig. 1. Adversarial Network Compression: Our method consists of the teacher, student and discriminator networks. The teacher is trained in advance and used for supervision during adversarial learning, while the student and discriminator are both trainable. The discriminator takes as input the features from the teacher and student, as well as, the adversarial sample (i.e. student labeled as teacher). For the adversarial sample, we empirically found that dropout is beneficial. In addition, there is a L2 loss to force the student to mimic the output of the teacher.

\subsection{Generative Adversarial Networks}

We shortly discuss the Generative Adversarial Networks (GANs) 14 to illustrate the connection with our approach. In GANs, the main idea is to simultaneously train two networks (two-player game) that compete with each other in order to improve their objectives. The first network, the generator $G$, takes random noise (i.e. latent variables) input $z$ to generate images. In addition to the noise, the input can be conditioned on images or labels [36. In both cases, the goal is to learn generating images that look real by aligning the real data and model distributions. The second network, the discriminator $D$, takes as input an image from the data distribution and the generator's output; and the objective is to classify whether the image is real or fake. Overall, $G$ tries to fool $D$, while $D$ tries to detect input from $G$. Isola et al. 25] have shown that a conditional Generative Adversarial Network (cGAN) successfully transforms an input image to another modality $y$ using the adversarial learning. The objective of cGAN can be written as:

$$
\begin{aligned}
\mathcal{L}_{c G A N}(G, D)= & \mathbb{E}_{x, y \sim p_{\text {data }}(x, y)}[\log (D(x, y))]+ \\
& \mathbb{E}_{x \sim p_{\text {data }}(x), z \sim p_{z}(z)}[\log (1-D(x, G(x, z))],
\end{aligned}
$$

where $p_{\text {data }}(x, y)$ corresponds to the real data distribution over the input image $x$ and label $y$; and $p_{z}(z)$ to the prior distribution over the input noise $z$. During training, the objective is maximized w.r.t. $D$ and minimized w.r.t. $G$. In both cases, the loss is cross entropy for the binary $D$ output.

\subsection{Adversarial Compression}

In the context of network compression, we propose to adapt the two-player game based on the teacher and student. The goal now is to adversarially train $D$ 
to classify whether input samples come from the teacher or student network. Both networks share the same input, namely an image $x$, but their predictions differ. We choose $f_{t}^{k}(x)$ and $f_{s}^{l}(x)$ feature representations from both networks as input to $D$. During training, $D$ is firstly updated w.r.t the labels from both input samples (blue lines, Fig. 1). Next, the student network is updated by inverting the labels for the student samples (calling them teacher). The reason for changing the labels is to back-propagate gradients that guide the student to produce output as the teacher for the same input image (red line, Fig. 11). The teacher network has been trained in advance with labels and it is not updated during training. Eventually, fooling the discriminator translates in predicting the same output for teacher and student networks. This is the same objective as in network distillation. After reformulating Eq. (1), we define our objective as:

$$
\begin{aligned}
\mathcal{L}_{\text {Adv }}\left(f_{s}, D\right)= & \mathbb{E}_{f_{t}^{k}(x) \sim p_{\text {teacher }}(x)}\left[\log \left(D\left(f_{t}^{k}(x)\right)\right)\right]+ \\
& \mathbb{E}_{f_{s}^{l}(x) \sim p_{\text {student }}(x), z \sim p_{z}(z)}\left[\log \left(1-D\left(f_{s}^{l}(x)\right)\right)\right] .
\end{aligned}
$$

where $p_{\text {teacher }}(x)$ and $p_{\text {student }}(x)$ correspond to the teacher and student feature distribution. We provide the noise input in the form of dropout applied on the student, similar to 25]. However, the dropout is active only during the student update (red arrow in Fig. 11). We experimentally found that using the dropout only for the student update gives more stable results for our problem. We omit $z$ in $f_{s}^{l}(x)$ to keep the notation simple.

The reason for using the features $f_{t}^{k}(x)$ and $f_{s}^{l}(x)$ as input to $D$, instead of the logits $f_{t}(x)$ and $f_{s}(x)$, is their dimensionality. The features usually have higher dimensions, which makes the judgment of the discriminator more challenging. We evaluate this statement later in the experimental section. Training $D$ with input from intermediate output $\left(f_{t}^{k}(x)\right.$ and $\left.f_{s}^{l}(x)\right)$ from teacher and student works fine for updating the parameters of $D$ and partially for the student. In student, there is a number of parameters until the final output $f_{s}(x)$ (logits) which also has to be updated. To address this problem, we seek to minimize the difference between the two networks output, namely $f_{t}(x)$ and $f_{s}(x)$. This is the data objective in our formulation that is given by:

$$
\mathcal{L}_{\text {Data }}\left(f_{s}\right)=\mathbb{E}_{f_{s}(x) \sim p_{\text {student }}(x)}\left[\left\|f_{t}(x)-f_{s}(x)\right\|_{2}^{2}\right] .
$$

The data term contributes to the update of all student parameters. We found it very important for the student network convergence (green dashed lines, Fig. 1) since our final goal is to match the output between the teacher and student. The final objective with both terms is expressed as:

$$
\arg \min _{f_{s}} \max _{D} \mathcal{L}_{A d v}\left(f_{s}, D\right)+\lambda \mathcal{L}_{\text {Data }}\left(f_{s}\right)
$$

where $\lambda$ is a tuning constant between the two terms.

The data term of our model is the same with the compression objective of $\mathrm{Ba}$ and Caruana [2] and in accordance with the work of Isola et al [25]. In addition, we aim for the exact output between teacher - student and thus using only the 
adversarial objective does not force the student to be as close as possible to the teacher. Note also that the role of $G$ is implicitly assigned to the student, but it is not explicitly required in our approach. For the adversarial part, we share the label inversion idea for the adversarial samples from ADDA [52] and reversal gradient [12. Below, we discuss the network architectures for exploring the idea of adversarial learning in network compression.

\subsection{Network Architectures}

Our model is composed of three networks: the teacher, student and discriminator $D$. Here, we present all three architectures.

Teacher We choose the latest version of ResNet [19] for this role, since it is currently the standard architecture for recognition tasks. The network has adaptive capacity based on the number of bottlenecks and number of feature per bottleneck. We select ResNet-164 for our experiments. To examine the generalization of our approach on small-scale experiments, the Network in Network $(\mathrm{NiN})[34$ is also selected as teacher. The teacher is trained in advance with labeled data using cross-entropy.

Student We found it meaningful to choose ResNet architecture for the student too. Although, the student is based on the same architecture, it has limited capacity. We perform our experiments with ResNet-18 and ResNet-20. For small-scale experiments, we employ LeNet-4 [30] for the student role. It is a shallow network and we experimentally found that it can be easily paired with NiN. The student network parameters are not trained on the labeled data. Furthermore, the labels are not used in the adversarial compression.

Discriminator This discriminator $D$ plays the most important role among the others. It can be interpreted as a loss function with parameters. The discriminator has to strike a balance between simplicity and network capacity to avoid being trivially fooled. We choose empirically a relative architecture. Our network is composed of three fully-connected (FC) layers (128 - 256 -128) where the network input comes from the teacher $f_{t}^{k}(x)$ and student $f_{s}^{l}(x)$. The intermediate activations are non-linearities (ReLUs). The output is a binary prediction, given by a sigmoid function. The network is trained with cross entropy where the objective is to predict between teacher or student. This architecture has been chosen among others, which we present in the experimental part (Sec. 4.1). Similar architectures are also maintained by [52] and [62] for adversarial learning.

\subsection{Discriminator Regularization}

The input to the discriminator has significantly lower dimensions in our problem compared to GANs for image generation [25[45]. As a result, it is simpler for the discriminator to understand the source of input. In particular, it can easily distinguish teacher from student samples from the early training stages (as also maintained in [14]). To address this limitation, we explore different ways of regularizing the discriminator. Our goal is to prevent the discriminator 
from dominating the training, while retaining the network capacity. We consider therefore three types of regularization, which we examine in our experiments.

L2 regularization This is the standard way of regularizing a neural network [39]. At first, we try the L2 regularization to force the weights of the discriminator not to grow. The term is given by:

$$
\mathcal{L}_{\text {regul }}(D)=-\mu \sum_{i=1}^{n}\left\|w_{D, i}\right\|_{2}^{2}
$$

where $n$ is the number of network parameters and $\mu$ controls the contribution of the regularizer to the optimization. The parameters of $D$ correspond to $w_{D, i}$.

L1 regularization Additionally, we try L1 regularization which supports sparse weights. This is formalized as:

$$
\mathcal{L}_{\text {regul }}(D)=-\mu \sum_{i=1}^{n}\left|w_{D, i}\right| .
$$

In both Eq. (5) and (6) there is negative sign, because the term is updated during the maximization step of Eq. (4).

Adversarial samples for $\boldsymbol{D}$ In the above cases, there is no guarantee that the discriminator will become weaker w.r.t student. We propose to achieve it by updating $D$ with adversarial samples. According to the objective in Eq. (4), the discriminator is updated only with correct labels. Here, we additionally update $D$ with student samples that are labeled as teacher. This means that we use the same adversarial samples to update both student and $D$. The new regularizer is defined as:

$$
\mathcal{L}_{\text {regul }}(D)=\mathbb{E}_{f_{s}^{l}(x) \sim p_{\text {student }}(x)}\left[\log D\left(f_{s}^{l}(x)\right)\right] .
$$

The motivation behind the regularizer is to prolong the game between the student and discriminator. Eventually, the discriminator manages to distinguish teacher and student samples, as we have observed. However, the longer it takes the discriminator to win the game, the more valuable gradient updates the student receives. The same principle has been also explored for text synthesis [42. Applying the same idea on the teacher samples does not hold, since it is fixed and thus a reference in training. Our objective now becomes:

$$
\arg \min _{f_{s}} \max _{D} \mathcal{L}_{A d v}\left(f_{s}, D\right)+\lambda \mathcal{L}_{\text {Data }}\left(f_{s}\right)+\mathcal{L}_{\text {regul }}(D)
$$

where the regularization $\mathcal{L}_{\text {regul }}(D)$ corresponds to one of the above approaches. In the experimental section, we show that our method requires the regularization in order to achieve good results. In addition, we observed that the introduced regularization had the most significant influence in fooling $D$, since it is conditioned on the student. 


\subsection{Learning \& Optimization}

The network compression occurs in two phases. First, the teacher is trained from scratch on labeled data. Second, the student is trained together with $D$. The student is randomly initialized, as well as, $D$. All models are trained using Stochastic Gradient Descent (SGD) with momentum. The learning rate is 0.001 for the first $80 k$ training iterations and then it is decreased by one magnitude. The weight decay is set to 0.0002 . The min-batch size is to 128 samples. Furthermore, the dropout is set to 0.5 for the adversarial sample input to $D$. To further regularize the data, data augmentation (random crop and flip) is also included in training. In all experiments, the mean of the training set images is subtracted and they are divided by the standard deviation. Lastly, different weighting factors $\lambda$ have been examined, but we concluded that equal weighting is a good compromise for all evaluations. In the L1/L2 regularization, the value of $\mu$ is set to 0.99 . The same protocol is followed for all datasets, unless it is differently stated.

\section{Evaluation}

In this section, we evaluate our approach on five standard classification datasets: CIFAR-10 [27, CIFAR-100 [27, SVHN [37, Fashion-MNIST [55] and ImageNet 2012 [10]. In total, we examine three teacher and three student architectures.

Our ultimate goal is to train the shallower and faster student network to perform, at the level of accuracy, as close as possible to the deeper and complex teacher. Secondly, we aim to outperform the student trained with supervision by transferring knowledge from the teacher. We report therefore for each experiment the error, numbers of parameters and floating point operations (FLOPs). The last two metrics are reported in M-Million or B-Billion scale.

Once we choose the discriminator $D$ and regularization in Sec. 4.1, we perform a set of baseline evaluations and comparisons with related approaches for all datasets in Sec. 4.2 and Sec. 4.3 .

Implementation details Our implementation is based on TensorFlow [1. We also rely on our own implementation for the approach of Ba and Caruana 2] and Hinton et al. [20]. The results of the other approaches are obtained from the respective publications. Regarding the network architectures, we rely on the official TF code for all ResNet variants, while we implement by ourselves the Network in Network (NiN) and LeNet-4 models.

\subsection{Discriminator Model}

We discuss the choice of the discriminator $D$ architecture and the impact of the regularization on $D$.

Architecture We examine which $D$ architecture should be considered for adversarial compression. To this end, we consider CIFAR-100 dataset as the most representative among the small-scale datasets and train our student with adversarial compression. Since the role of the discriminator would be to ensure the 
Table 1. Discriminator Evaluation We choose the discriminator which enables the best student performance on CIFAR-100, when integrated in the proposed adversarial compression framework. Fully connected (fc) and convolutional (conv) layers are examined. We report the student classification error. The best performing model (128fc - 256fc - 128fc) is used in all other evaluations.

\begin{tabular}{l|c|l|c}
\hline Architecture & Error[\%] & Architecture & Error[\%] \\
\hline $\mathbf{1 2 8 f c}-\mathbf{2 5 6 f c}-\mathbf{1 2 8 f c}$ & $\mathbf{3 2 . 4 5}$ & $500 f c-500 f c$ & 33.28 \\
$64 f c-128 f c-256 f c$ & 32.78 & $256 f c-256 f c-64 f c$ & 33.46 \\
$256 f c-256 f c$ & 32.82 & $64 f c-64 f c$ & 33.51 \\
$256 f c-128 f c-64 f c$ & 33.05 & $128 c o n v-256 c o n v$ & 33.68 \\
$64 f c-128 f c-128 f c-64 f c$ & 33.09 & $128 f c-128 f c-128 f c$ & 33.72
\end{tabular}

best student training, we explore several architectures and select the one that is providing the minimum classification error of the trained student. The discriminator models, except one, are fully connected (fc) with (ReLU) activation, other than the last layer. We explore two to four fc-layer models with different capacity. We also made experiments with a convolutional (conv) discriminator which has lower performance than fc discriminators. The results for the discriminator trials are in Table 1 . The best architecture is given by 3 fc-layers of depth 128-256-128. Notice that our best architecture is similar to the $D$ models for adversarial domain adaption 52 and perceptual similarity 62 .

Regularization Here we experiment on the three regularization techniques, described in Sec. 3.5, on four datasets. We rely on our best performing model, i.e. the one with features provided as input to $D$ and dropout on the student. The results are summarized in Table 2. The lack of regularization leads to poorer performance since it is more difficult to fool the discriminator based on our observations. In particular, the performance without regularization is worse than training the student architecture on supervised learning as we show in Sec. 4.2. Adding the L1 or L2 regularizer indicates an important error drop (L1 and L2 column in Table 2. However, our proposed regularization introduces the most difficulties in the discriminator that leads to better performance. We use the adversarial samples for $D$ regularization for the rest evaluations.

\subsection{Component Evaluation}

Initially, the teacher is trained with labels (i.e. supervised teacher in Table 3 6). Next, the adversarial compression is performed under different configurations. The input to $D$ is either the logits or features. In both cases, we also examine the effect of the dropout on the student. In all experiments, we train the student only based on teacher supervision and without labels. The results for every experiment are reported in Table 3.6. In all baselines, there is the $L 2$ loss on the logits from the teacher and student (i.e. $f_{t}(x)$ and $\left.f_{s}(x)\right)$. We also provide the results of the same network as the student trained with labels (i.e. supervised student in Table 3,6 . 
Table 2. Regularization Evaluation We evaluate three different ways of regularizing the discriminator $D$. We also show the performance without regularization. The error is in percentage. Our adversarial sample in $D$ regularization is presented in the last column. All experiments have been accomplished with our complete model, namely features input to $D$ and dropout on the student.

\begin{tabular}{l|c|c|c|c|c|c} 
Dataset & Teacher & Student & W/o Regul. & L1 & L2 & Ours \\
\hline CIFAR-10 & ResNet-164 & ResNet-20 & 10.07 & 8.19 & 8.16 & 8.08 \\
CIFAR-100 & ResNet-164 & ResNet-20 & 34.10 & 33.36 & 33.02 & 32.45 \\
SVHN & ResNet-164 & ResNet-20 & 3.73 & 3.67 & 3.68 & 3.66 \\
F-MNIST & NiN & LeNet-4 & 9.62 & 8.91 & 8.75 & 8.64
\end{tabular}

On CIFAR-10, CIFAR-100 and SVHN experiments, the input to $D$ from ResNet-164 and ResNet-20 is the features of the average pool layer, which are used for teacher $f_{t}^{k}(x)$ and student $f_{s}^{l}(x)$. On Fashion-MNIST, it is the output of the last fully connected layer before the logits both for $\mathrm{NiN}$ (teacher) and LeNet-4 (student). The model training runs for 260 epochs in CIFAR-10, CIFAR100 and SVHN, while for 120 epochs in Fashion-MNIST. Below, the results are individually discussed for each dataset.

Table 3. CIFAR-10 Evaluation We evaluate the components of our approach. ResNet-164 Parameters: 2.6M, FLOPs: 97.49B. ResNet-20 Parameters: 0.27M, FLOPs: 10.52B. Our student, ResNet20 , has around $10 \mathrm{x}$ less parameters.

\begin{tabular}{l|c}
\hline Model & Error[\%] \\
\hline $\begin{array}{l}\text { Supervised teacher } \\
\text { ResNet-164 }\end{array}$ & 6.57 \\
\hline Supervised student & 8.58 \\
ResNet-20 & \\
\hline Our student ( $D$ with logits) & 8.31 \\
$\quad+$ dropout on student & 8.10 \\
Our student ( $D$ with features) & 8.10 \\
$\quad+$ dropout on student & 8.08
\end{tabular}

Table 4. CIFAR-100 Evaluation The component evaluation is presented. We use the same teacher and student models as in CIFAR-10. ResNet-164 Parameters: 2.6M, FLOPs: 97.49B. ResNet-20 Parameters: 0.27M, FLOPs: 10.52B.

\begin{tabular}{l|c}
\hline Model & Error[\%] \\
\hline $\begin{array}{l}\text { Supervised teacher } \\
\text { ResNet-164 }\end{array}$ & 27.76 \\
\hline Supervised student & 33.36 \\
ResNet-20 & \\
\hline Our student ( D with logits) & 33.96 \\
$\quad+$ dropout on student & 33.41 \\
Our student ( D with features) & 33.40 \\
$\quad+$ dropout on student & 32.45
\end{tabular}

CIFAR-10, Table 3. All compression baselines, based on student with ResNet-20, have only around $1.5 \%$ drop in performance compared to the teacher (ResNet-164). Moreover, they are all better than the student network, trained with supervision (i.e. labels), which is $2 \%$ behind the teacher. Our complete model benefits from the dropout on the adversarial samples and achieves the best performance using feature input to $D$.

CIFAR-100, Table 4. We also use Resnet-164 for teacher and ResNet20 for student to have 10x less parameters as in CIFAR-10. In this evaluation, the performance drop between the teacher and the compressed models is slightly 
Table 5. SVHN Evaluation We evaluate the components of our approach. ResNet-164 Parameters: 2.6M, FLOPs: 97.49B. ResNet-20 Parameters: 0.27M, FLOPs: 10.52B. The teacher and student model are similar to CIFAR evaluation.

\begin{tabular}{l|c}
\hline Model & Error[\%] \\
\hline $\begin{array}{l}\text { Supervised teacher } \\
\text { ResNet-164 }\end{array}$ & 3.98 \\
\hline Supervised student & 4.20 \\
ResNet-20 & \\
\hline Our student (D with logits) & 3.74 \\
$\quad+$ dropout on student & 3.81 \\
Our student (D with features) & 3.74 \\
$\quad+$ dropout on student & 3.66
\end{tabular}

Table 6. Fashion-MNIST Evaluation We evaluate the components with different teacher and student. NiN Parameters: 10.6M, FLOPs: 60.23B. LeNet-4 Parameters: 2.3M, FLOPs: 7.06B. Our student, LeNet-4, has around 5x less parameters.

\begin{tabular}{l|c}
\hline Model & Error[\%] \\
\hline Supervised teacher & 7.98 \\
NiN & \\
\hline Supervised student & 8.77 \\
LeNet-4 & \\
\hline Our student ( $D$ with logits) & 8.90 \\
$\quad+$ dropout on student & 8.84 \\
Our student ( $D$ with features) & 8.86 \\
$\quad+$ dropout on student & 8.61
\end{tabular}

Table 7. CIFAR-10 and CIFAR-100 Comparisons We compare our results and number of network parameters with related methods on similar architectures. We use ResNet-20 for our student and our complete model.

\begin{tabular}{c|c||c||c|c|c} 
CIFAR-10 & Error[\%] & Param. & CIFAR-100 & Error[\%] & Param. \\
\hline L2 - Ba et al. [2] & 9.07 & $0.27 \mathrm{M}$ & Yim et al. [59] & 36.67 & - \\
Hinton et al. [20] & 8.88 & $0.27 \mathrm{M}$ & FitNets [44] & 35.04 & $2.50 \mathrm{M}$ \\
Quantization [65] & 8.87 & $0.27 \mathrm{M}$ & Hinton et al. [20] & 33.34 & $0.27 \mathrm{M}$ \\
FitNets [4] & 8.39 & $2.50 \mathrm{M}$ & L2 - Ba et al. [2] & 32.79 & $0.27 \mathrm{M}$ \\
Binary Connect [9] & 8.27 & $15.20 \mathrm{M}$ & Our student & $\mathbf{3 2 . 4 5}$ & $0.27 \mathrm{M}$ \\
Yim et al. [59] & 11.30 & - & & & \\
Our student & $\mathbf{8 . 0 8}$ & $0.27 \mathrm{M}$ & & &
\end{tabular}

larger. The overall behavior is similar to CIFAR-10. However, the error is reduced by $1 \%$ after adding the dropout to the student using features as input to $D$. Here, we had the biggest improvement after using dropout.

SVHN, Table 5. In this experiment, the teacher and student architectures are still the same. Although, we tried the Network in Network (NiN) and LeNet4 as teacher and student, the pair did not perform as well as ResNet. Unlike in the previous experiments, here the Adam optimizer was used, as it improved across all ablation results. Notice that our student achieves better performance not only from the same network trained with labels, the supervised student, but from the teacher too. This is a known positive side product of the distillation 2 .

Fashion-MNIST, Table 6. We select Network in Network (NiN) as teacher and LeNet-4 as student. The dataset is relative simple and thus a ResNet architecture is not necessary. All approaches are close to each other.It is clear that the features input to $D$ and the dropout are important to obtain the best performance in comparison to the other baselines. For instance, the student network trained with supervision (error 8.77\%) is better than our baselines other than our complete model (error $8.61 \%$ ). Finally the Adam optimizer has been used. 
Table 8. SVHN and F-MNIST Comparisons We compare our results and number of network parameters with related methods on the same student architecture that is ResNet-20 for SVHN and LeNet-4 for Fashion-MNIST.

\begin{tabular}{c|c|c||c|c|c} 
SVHN & Error[\%] & Param. & F-MNIST & Error[\%] & Param. \\
\hline L2 - Ba et al. [2] & 3.75 & $0.27 \mathrm{M}$ & L2 - Ba et al. [2] & 8.89 & $2.3 \mathrm{M}$ \\
Hinton et al. [20] & 3.66 & $0.27 \mathrm{M}$ & Hinton et al. 20] & 8.71 & $2.3 \mathrm{M}$ \\
Our student & $\mathbf{3 . 6 6}$ & $0.27 \mathrm{M}$ & Our student & $\mathbf{8 . 6 4}$ & $2.3 \mathrm{M}$
\end{tabular}

Common conclusions There is a number of common outcomes for all evaluations: 1. the adversarial compression reaches the lowest error when using features as input to $D ; 2$. our student performs always better than training the same network with labels (i.e. supervised student) and 3. we achieve good generalization on different teacher - student architectures.

Comparisons to state-of-the-art In Tables 7 and 8, we compare our student with other compression strategies on CIFAR-10 and CIFAR-100. We choose four distillation [2 2044/59] and two quantization 965] approaches for CIFAR-10. We examine the same four distillation methods for a comparison on CIFAR-100. The work of Ba and Caruana 2] is closer to our approach, because it relies on L2 minimization, though it is on the logits (see Table 7). The Knowledge Distillation (KD) [20] is also related to our idea, but it relies on labels. Both evaluations demonstrate that we achieve the lowest error and our student has the smallest number of parameters. In addition, we compare our results on SVHN and Fashion-MNIST with two distillation approaches (see Table 8). The error here is much lower for methods, but we are consistently better than the other approaches. Next, We demonstrate the same findings on large-scale experiments.

\subsection{ImageNet Evaluation}

We perform an evaluation on ImageNet to examine whether the distillation is possible on a large-scale dataset with class number set to 1000 . The teacher is a pre-trained ResNet-152, while we try two different student architectures. At first, we choose ResNet-18 to train our student using features as input to $D$ and adding the dropout on the adversarial samples. Regarding the experimental settings, we have set the batch size to 256 , while the rest hyper-parameters remain the same. All networks use the the average pool layer to output features for $D$. We evaluate on the validation dataset. The results are presented in Table 9.

Our findings are consistent with the earlier evaluations. Our best performing model (features input to $D$ and dropout on student) perform at best and better than the student trained with supervision. Secondly, we examine a stronger student where we employ ResNet-50 for training our model. We present our results in Table 10 where we also compare with binarization, distillation and factorization methods. Although we achieve the best results, MobileNets has fewer parameters. We see the adversarial network compression on MobileNets as future work. 
Table 9. ImageNet Baselines We evaluate the components of our approach. ResNet152 Parameters: 58.21M, FLOPs: 5587B. ResNet-18 Parameters: 13.95M, FLOPs: 883.73B. Our student has around $4 \mathrm{x}$ less parameters. Our model has features as input to $D$ and dropout on the adversarial samples.

\begin{tabular}{l|cc}
\hline Model & Top-1 & Top-5 \\
Error[\%] & Error[\%] \\
\hline Supervised teacher (ResNet-152) & 27.63 & 5.90 \\
Supervised student (ResNet-18) & 43.33 & 20.11 \\
\hline Our student (D with features) & 33.31 & 11.96 \\
$\quad+$ dropout on student & $\mathbf{3 2 . 8 9}$ & $\mathbf{1 1 . 7 2}$
\end{tabular}

Table 10. ImageNet Evaluation We evaluate two versions of our student and compare with related methods. ResNet-152 Parameters: 58.21M, FLOPs: 5587B. ResNet50 Parameters: 37.49M, FLOPs: 2667B. Our student, ResNet-50, has around 2x less parameters. We also include the student ResNet-18 from the evaluation in Table 9. Our student is trained with features as input to $D$ and dropout on the student.

\begin{tabular}{l|ccc}
\hline Model & Top-1 Error[\%] & Top-5 Error[\%] & Parameters \\
Supervised teacher (ResNet-152) & 27.63 & 5.90 & $58.21 \mathrm{M}$ \\
Supervised student (ResNet-50) & 30.30 & 10.61 & $37.49 \mathrm{M}$ \\
\hline XNOR [1] (ResNet-18) & 48.80 & 26.80 & $13.95 \mathrm{M}$ \\
Binary-Weight [4] (ResNet-18) & 39.20 & 17.00 & $13.95 \mathrm{M}$ \\
L2 - Ba et al. [2] (ResNet-18) & 33.28 & 11.86 & $13.95 \mathrm{M}$ \\
MobileNets [21] & 29.27 & 10.51 & $4.20 \mathrm{M}$ \\
L2 - Ba et al. [2] (ResNet-50) & 27.99 & 9.46 & $37.49 \mathrm{M}$ \\
Our student (ResNet-18) & 32.89 & 11.72 & $13.95 \mathrm{M}$ \\
Our student (ResNet-50) & $\mathbf{2 7 . 4 8}$ & $\mathbf{8 . 7 5}$ & $37.49 \mathrm{M}$
\end{tabular}

\section{Conclusion}

We have presented the adversarial network compression for knowledge transfer between a large model and a smaller one with limited capacity. We have empirically shown that regularization helps the student to compete with the discriminator. Finally, we show state-of-the-art performance without using labels in an extensive evaluation of five datasets, three teacher and three student architectures. As future work, we aim to explore adversarial schemes with more discriminators that use intermediate feature representations, as well as, transferring our approach to different tasks such as object detection and segmentation.

\section{Acknowledgements}

This research was partially supported by BMWi - Federal Ministry for Economic Affairs and Energy (MEC-View Project). 


\section{References}

1. Abadi, M., Barham, P., Chen, J., Chen, Z., Davis, A., Dean, J., Devin, M., Ghemawat, S., Irving, G., Isard, M., et al.: Tensorflow: A system for large-scale machine learning. In: OSDI. vol. 16, pp. 265-283 (2016)

2. Ba, J., Caruana, R.: Do deep nets really need to be deep? In: Advances in neural information processing systems. pp. 2654-2662 (2014)

3. Belagiannis, V., Zisserman, A.: Recurrent human pose estimation. In: Automatic Face \& Gesture Recognition (FG 2017), 2017 12th IEEE International Conference on. pp. 468-475. IEEE (2017)

4. Bucilu, C., Caruana, R., Niculescu-Mizil, A.: Model compression. In: Proceedings of the 12th ACM SIGKDD international conference on Knowledge discovery and data mining. pp. 535-541. ACM (2006)

5. Carreira-Perpinán, M.A., Idelbayev, Y.: learning-compression algorithms for neural net pruning. In: Proceedings of the IEEE Conference on Computer Vision and Pattern Recognition. pp. 8532-8541 (2018)

6. Chen, L.C., Papandreou, G., Kokkinos, I., Murphy, K., Yuille, A.L.: Deeplab: Semantic image segmentation with deep convolutional nets, atrous convolution, and fully connected crfs. arXiv preprint arXiv:1606.00915 (2016)

7. Chen, W., Wilson, J., Tyree, S., Weinberger, K., Chen, Y.: Compressing neural networks with the hashing trick. In: International Conference on Machine Learning. pp. 2285-2294 (2015)

8. Chollet, F.: Xception: Deep learning with depthwise separable convolutions. arXiv preprint arXiv:1610.02357 (2016)

9. Courbariaux, M., Bengio, Y., David, J.P.: Binaryconnect: Training deep neural networks with binary weights during propagations. In: Advances in Neural Information Processing Systems. pp. 3123-3131 (2015)

10. Deng, J., Dong, W., Socher, R., Li, L.J., Li, K., Fei-Fei, L.: Imagenet: A large-scale hierarchical image database. In: Computer Vision and Pattern Recognition, 2009. CVPR 2009. IEEE Conference on. pp. 248-255. IEEE (2009)

11. Denton, E.L., Zaremba, W., Bruna, J., LeCun, Y., Fergus, R.: Exploiting linear structure within convolutional networks for efficient evaluation. In: Advances in Neural Information Processing Systems. pp. 1269-1277 (2014)

12. Ganin, Y., Lempitsky, V.: Unsupervised domain adaptation by backpropagation. In: International Conference on Machine Learning. pp. 1180-1189 (2015)

13. Gong, Y., Liu, L., Yang, M., Bourdev, L.: Compressing deep convolutional networks using vector quantization. arXiv preprint arXiv:1412.6115 (2014)

14. Goodfellow, I., Pouget-Abadie, J., Mirza, M., Xu, B., Warde-Farley, D., Ozair, S., Courville, A., Bengio, Y.: Generative adversarial nets. In: Advances in neural information processing systems. pp. 2672-2680 (2014)

15. Han, S., Mao, H., Dally, W.J.: Deep compression: Compressing deep neural networks with pruning, trained quantization and huffman coding. arXiv preprint arXiv:1510.00149 (2015)

16. Han, S., Pool, J., Tran, J., Dally, W.: Learning both weights and connections for efficient neural network. In: Advances in Neural Information Processing Systems. pp. 1135-1143 (2015)

17. Hassibi, B., Stork, D.G.: Second order derivatives for network pruning: Optimal brain surgeon. In: Advances in neural information processing systems. pp. 164-171 (1993) 
18. He, K., Zhang, X., Ren, S., Sun, J.: Deep residual learning for image recognition. In: Proceedings of the IEEE conference on computer vision and pattern recognition. pp. 770-778 (2016)

19. He, K., Zhang, X., Ren, S., Sun, J.: Identity mappings in deep residual networks. In: European Conference on Computer Vision. pp. 630-645. Springer (2016)

20. Hinton, G., Vinyals, O., Dean, J.: Distilling the knowledge in a neural network. arXiv preprint arXiv:1503.02531 (2015)

21. Howard, A.G., Zhu, M., Chen, B., Kalenichenko, D., Wang, W., Weyand, T., Andreetto, M., Adam, H.: Mobilenets: Efficient convolutional neural networks for mobile vision applications. arXiv preprint arXiv:1704.04861 (2017)

22. Huang, G., Liu, S., van der Maaten, L., Weinberger, K.Q.: Condensenet: An efficient densenet using learned group convolutions. group 3(12), 11 (2017)

23. Huang, G., Sun, Y., Liu, Z., Sedra, D., Weinberger, K.Q.: Deep networks with stochastic depth. In: European Conference on Computer Vision. pp. 646-661. Springer (2016)

24. Iandola, F.N., Han, S., Moskewicz, M.W., Ashraf, K., Dally, W.J., Keutzer, K.: Squeezenet: Alexnet-level accuracy with 50x fewer parameters andi $0.5 \mathrm{mb}$ model size. arXiv preprint arXiv:1602.07360 (2016)

25. Isola, P., Zhu, J.Y., Zhou, T., Efros, A.A.: Image-to-image translation with conditional adversarial networks. arXiv preprint arXiv:1611.07004 (2016)

26. Jaderberg, M., Vedaldi, A., Zisserman, A.: Speeding up convolutional neural networks with low rank expansions. arXiv preprint arXiv:1405.3866 (2014)

27. Krizhevsky, A., Hinton, G.: Learning multiple layers of features from tiny images (2009)

28. Krizhevsky, A., Sutskever, I., Hinton, G.E.: Imagenet classification with deep convolutional neural networks. In: Advances in neural information processing systems. pp. 1097-1105 (2012)

29. Lebedev, V., Ganin, Y., Rakhuba, M., Oseledets, I., Lempitsky, V.: Speeding-up convolutional neural networks using fine-tuned cp-decomposition. arXiv preprint arXiv:1412.6553 (2014)

30. LeCun, Y., Boser, B.E., Denker, J.S., Henderson, D., Howard, R.E., Hubbard, W.E., Jackel, L.D.: Handwritten digit recognition with a back-propagation network. In: Advances in neural information processing systems. pp. 396-404 (1990)

31. Li, F., Zhang, B., Liu, B.: Ternary weight networks. arXiv preprint arXiv:1605.04711 (2016)

32. Li, H., Kadav, A., Durdanovic, I., Samet, H., Graf, H.P.: Pruning filters for efficient convnets. arXiv preprint arXiv:1608.08710 (2016)

33. Li, J., Zhao, R., Huang, J.T., Gong, Y.: Learning small-size dnn with outputdistribution-based criteria. In: Fifteenth Annual Conference of the International Speech Communication Association (2014)

34. Lin, M., Chen, Q., Yan, S.: Network in network. arXiv preprint arXiv:1312.4400 (2013)

35. Lin, T.Y., Goyal, P., Girshick, R., He, K., Dollár, P.: Focal loss for dense object detection. In: Proceedings of the IEEE International Conference on Computer Vision (2017)

36. Mirza, M., Osindero, S.: Conditional generative adversarial nets (2014)

37. Netzer, Y., Wang, T., Coates, A., Bissacco, A., Wu, B., Ng, A.Y.: Reading digits in natural images with unsupervised feature learning. In: NIPS Workshop on Deep Learning and Unsupervised Feature Learning 2011 (2011) 
38. Newell, A., Yang, K., Deng, J.: Stacked hourglass networks for human pose estimation. In: European Conference on Computer Vision. pp. 483-499. Springer (2016)

39. Ng, A.Y.: Feature selection, 11 vs. 12 regularization, and rotational invariance. In: Proceedings of the twenty-first international conference on Machine learning. p. 78. ACM (2004)

40. Polino, A., Pascanu, R., Alistarh, D.: Model compression via distillation and quantization. arXiv preprint arXiv:1802.05668 (2018)

41. Rastegari, M., Ordonez, V., Redmon, J., Farhadi, A.: Xnor-net: Imagenet classification using binary convolutional neural networks. In: European Conference on Computer Vision. pp. 525-542. Springer (2016)

42. Reed, S., Akata, Z., Yan, X., Logeswaran, L., Schiele, B., Lee, H.: Generative adversarial text to image synthesis. arXiv preprint arXiv:1605.05396 (2016)

43. Rigamonti, R., Sironi, A., Lepetit, V., Fua, P.: Learning separable filters. In: Proceedings of the IEEE Conference on Computer Vision and Pattern Recognition. pp. 2754-2761 (2013)

44. Romero, A., Ballas, N., Kahou, S.E., Chassang, A., Gatta, C., Bengio, Y.: Fitnets: Hints for thin deep nets. arXiv preprint arXiv:1412.6550 (2014)

45. Salimans, T., Goodfellow, I., Zaremba, W., Cheung, V., Radford, A., Chen, X.: Improved techniques for training gans. In: Advances in Neural Information Processing Systems. pp. 2234-2242 (2016)

46. Schmidhuber, J.: Learning complex, extended sequences using the principle of history compression. Neural Computation 4(2), 234-242 (1992)

47. Simonyan, K., Zisserman, A.: Very deep convolutional networks for large-scale image recognition. arXiv preprint arXiv:1409.1556 (2014)

48. Soudry, D., Hubara, I., Meir, R.: Expectation backpropagation: Parameter-free training of multilayer neural networks with continuous or discrete weights. In: Advances in Neural Information Processing Systems. pp. 963-971 (2014)

49. Ström, N.: Phoneme probability estimation with dynamic sparsely connected artificial neural networks. The Free Speech Journal 5, 1-41 (1997)

50. Szegedy, C., Vanhoucke, V., Ioffe, S., Shlens, J., Wojna, Z.: Rethinking the inception architecture for computer vision. In: Proceedings of the IEEE Conference on Computer Vision and Pattern Recognition. pp. 2818-2826 (2016)

51. Tung, F., Mori, G.: Clip-q: Deep network compression learning by in-parallel pruning-quantization. In: Proceedings of the IEEE Conference on Computer Vision and Pattern Recognition. pp. 7873-7882 (2018)

52. Tzeng, E., Hoffman, J., Saenko, K., Darrell, T.: Adversarial discriminative domain adaptation. arXiv preprint arXiv:1702.05464 (2017)

53. Urban, G., Geras, K.J., Kahou, S.E., Aslan, O., Wang, S., Caruana, R., Mohamed, A., Philipose, M., Richardson, M.: Do deep convolutional nets really need to be deep and convolutional? arXiv preprint arXiv:1603.05691 (2016)

54. Wu, J., Leng, C., Wang, Y., Hu, Q., Cheng, J.: Quantized convolutional neural networks for mobile devices. In: Proceedings of the IEEE Conference on Computer Vision and Pattern Recognition. pp. 4820-4828 (2016)

55. Xiao, H., Rasul, K., Vollgraf, R.: Fashion-mnist: a novel image dataset for benchmarking machine learning algorithms. arXiv preprint arXiv:1708.07747 (2017)

56. Xie, S., Girshick, R., Dollár, P., Tu, Z., He, K.: Aggregated residual transformations for deep neural networks. arXiv preprint arXiv:1611.05431 (2016)

57. Xu, Z., Hsu, Y.C., Huang, J.: Training student networks for acceleration with conditional adversarial networks. In: BMVC. British Machine Vision Association (2018) 
58. Yang, Z., Moczulski, M., Denil, M., de Freitas, N., Smola, A., Song, L., Wang, Z.: Deep fried convnets. In: Proceedings of the IEEE International Conference on Computer Vision. pp. 1476-1483 (2015)

59. Yim, J., Joo, D., Bae, J., Kim, J.: A gift from knowledge distillation: Fast optimization, network minimization and transfer learning. In: The IEEE Conference on Computer Vision and Pattern Recognition (CVPR) (2017)

60. Yu, R., Li, A., Chen, C.F., Lai, J.H., Morariu, V.I., Han, X., Gao, M., Lin, C.Y., Davis, L.S.: Nisp: Pruning networks using neuron importance score propagation. Preprint at https://arxiv. org/abs/1711.05908 (2017)

61. Zagoruyko, S., Komodakis, N.: Wide residual networks. arXiv preprint arXiv:1605.07146 (2016)

62. Zhang, R., Isola, P., Efros, A.A., Shechtman, E., Wang, O.: The unreasonable effectiveness of deep features as a perceptual metric. arXiv preprint arXiv:1801.03924 (2018)

63. Zhang, X., Zhou, X., Lin, M., Sun, J.: Shufflenet: An extremely efficient convolutional neural network for mobile devices. arXiv preprint arXiv:1707.01083 (2017)

64. Zhou, A., Yao, A., Guo, Y., Xu, L., Chen, Y.: Incremental network quantization: Towards lossless cnns with low-precision weights (2017)

65. Zhu, C., Han, S., Mao, H., Dally, W.J.: Trained ternary quantization. arXiv preprint arXiv:1612.01064 (2016) 